\title{
On the Ramanujan AGM Fraction, II: The Complex-Parameter Case
}

\author{
J. Borwein and R. Crandall
}

\section{CONTENTS}

1. Background

2. The Instance $a^{2}=b^{2}$

3. Even/Odd Fractions

4. $\gamma$-Fractions

5. Divergence

6. AGM Relation Revisited

7. Open Issues

Acknowledgments

References

2000 AMS Subject Classification: Primary 44-A20; Secondary 33C05, 11J70

Keywords: Continued fractions, theta functions, elliptic integrals, hypergeometric functions, special functions, several complex variables
The Ramanujan continued fraction

$$
\mathcal{R}_{\eta}(a, b)=\frac{a}{\eta+\frac{b^{2}}{\eta+\frac{4 a^{2}}{\eta+\frac{9 b^{2}}{\eta+} \cdot}}}
$$

is interesting in many ways; e.g., for certain complex parameters $(\eta, a, b)$ one has an attractive $\mathrm{AGM}$ relation $\mathcal{R}_{\eta}(a, b)+$ $\mathcal{R}_{\eta}(b, a)=2 \mathcal{R}_{\eta}((a+b) / 2, \sqrt{a b})$. Alas, for some parameters the continued fraction $\mathcal{R}_{\eta}$ does not converge; moreover, there are converging instances where the AGM relation itself does not hold. To unravel these dilemmas we herein establish convergence theorems, the central result being that $\mathcal{R}_{1}$ converges whenever $|a| \neq|b|$. Such analysis leads naturally to the conjecture that divergence occurs whenever $a=b e^{i \phi}$ with $\cos ^{2} \phi \neq 1$ (which conjecture has been proven in a separate work) [Borwein et al. 04b.] We further conjecture that for $a / b$ lying in a certain-and rather picturesque-complex domain, we have both convergence and the truth of the AGM relation.

\section{BACKGROUND}

In a companion treatment [Borwein et al. 04a] we focused on evaluation of the continued fraction

$$
\mathcal{R}_{1}(a, b)=\frac{a}{1+\frac{b^{2}}{1+\frac{4 a^{2}}{1+\frac{9 b^{2}}{1+} \cdot}}}
$$

for real parameters $a$ and $b$. Note that, formally, $\mathcal{R}_{\eta}(a, b)=\mathcal{R}_{1}(a / \eta, b / \eta)$ so that with impunity we may focus upon the fraction displayed in the abstract, with $\eta:=1$; thus, we have a two-complex-parameter problem. For complex parameters $(a, b)$ convergence of $\mathcal{R}_{1}$ turns out to be - both historically and currently - problematic. 
A formal AGM relation - known to be true at least for positive real $a$ and $b$ [Borwein et al. 04a] - reads

$$
\mathcal{R}_{1}\left(\frac{a+b}{2}, \sqrt{a b}\right)=\frac{\mathcal{R}_{1}(a, b)+\mathcal{R}_{1}(b, a)}{2} .
$$

Yet, one wishes the three relevant fractions to converge prior to any resolution of the truth of such an AGM relation. So, we are primarily concerned with a precise determination of the convergence domain

$$
\mathcal{D}_{0}:=\left\{(a, b) \in \mathcal{C} \times \mathcal{C}: \mathcal{R}_{1}(a, b) \text { converges on } \hat{\mathcal{C}}\right\}
$$

where $\hat{\mathcal{C}}:=\mathcal{C} \cup\{\infty\}$ denotes the extended complex field. It is important to note what is meant by "convergence" on $\hat{\mathcal{C}}$ in the modern complex-continued-fraction context. If $p_{n} / q_{n}$ is the $n$th convergent to $\mathcal{R}_{1}$ (we remind ourselves in Section 3 of the definition of such convergents), we say that $\mathcal{R}_{1}$ converges if $p_{n} / q_{n}$ has a limit in $\hat{\mathcal{C}}$. Thus, divergence (nonconvergence) must be oscillatory - say bifurcated or chaotic (later, we exhibit examples of such divergence scenarios). This modern definition of convergence conveniently handles situations, such as the instance that $b^{2} /\left(1+4 a^{2} /+\ldots\right)$ converges to a value $(-1) \in \mathcal{C}$, whence $\mathcal{R}_{1}=\infty$ still converges on $\hat{\mathcal{C}}$.

Some preliminary nomenclature is relevant. We shall often refer to real cuts, that is sets $(\alpha, \beta)$ for reals $\alpha<\beta$; when we say a complex number $z$ belongs to $(\alpha, \beta)$, we mean $z$ must be real with $z \in(a, b)$ in the usual sense of real-interval membership. For example, $z$ is pure-imaginary-i.e., $z=0+i y$ with real $y \neq 0$-iff $z^{2} \in(-\infty, 0)$. Also the cut $(-\infty,-1 / 4)$ (and its closure $(-\infty,-1 / 4])$ will loom importantly in our convergence analysis.

We are eventually motivated to consider a special set $\mathcal{H}$ that turns out to be the open exterior of a cardioidknot (the picturesque character of $\mathcal{H}$ is exhibited in the companion treatment [Borwein et al. 04a]) as

$$
\mathcal{H}:=\{z \in \mathcal{C}:|\sqrt{z} /(1+z)|<1 / 2\}
$$

where we note for the moment that the classical AGM inequality $(a+b) / 2>\sqrt{a b}$ for positive real $a \neq b$ is true in the sense of magnitude-i.e., $|(a+b) / 2|>|\sqrt{a b}|$ - when $a / b \in \mathcal{H}$.

We next establish two-complex-parameter domain definitions

$$
\begin{gathered}
\mathcal{D}_{2}:=\{(a, b) \in \mathcal{C} \times \mathcal{C}:|a| \neq|b|\} \\
\mathcal{D}_{3}:=\left\{(a, b) \in \mathcal{C} \times \mathcal{C}: a^{2}=b^{2} \notin(-\infty, 0)\right\} \\
\mathcal{D}_{1}:=\mathcal{D}_{2} \cup \mathcal{D}_{3} .
\end{gathered}
$$

Our central result will be that

$$
\mathcal{D}_{1} \subseteq \mathcal{D}_{0}
$$

and we are eventually led to conjecture that, in fact, $\mathcal{D}_{1}=\mathcal{D}_{0}$, which would establish the precise convergence domain for $\mathcal{R}_{1}(a, b)$. As intimated in our abstract, this conjecture has been resolved in a separate treatment [Borwein et al. 04b] that employs apparatus from Section 5.

It is a tribute to the profundity of the Ramanujan construction that in the following treatment we need to rely upon some of the deepest theorems in complex-continued-fraction theory, including Stieltjesfraction theorems, convergence-set results such as the "parabola-sequence" and "oval" theorems, and yet other results from the finest of the complex-fraction literature.

\section{THE INSTANCE $a^{2}=b^{2}$}

Assume $a^{2}=b^{2}$. Clearly, if $\mathcal{R}_{1}(a, b)$ converges then, by the very definition of the $\mathcal{R}_{1}$ fraction, each of the four constructs $\mathcal{R}_{1}( \pm a, \pm b)$ converges (to $\left.\pm \mathcal{R}_{1}(a, b)\right)$. So, it suffices to analyze just

$$
\frac{a}{\mathcal{R}_{1}(a, a)}=1+\frac{\alpha_{1} z}{1+\frac{\alpha_{2} z}{1+\frac{\alpha_{3} z}{1+\frac{\alpha_{4} z}{1+}}}}=1+S(z)
$$

where $\alpha_{n}:=n^{2}, z:=a^{2}$, and $S$ is a classical Stieltjes fraction (as all $\alpha_{n}$ are positive real). We are led immediately to the following theorem:

Theorem 2.1. $a / \mathcal{R}_{1}(a, a)$ converges to a holomorphic function of $a^{2}$ on either half-plane $\operatorname{Re}(a)>0$ or $\operatorname{Re}(a)<$ 0 , and so, for $a^{2}=b^{2}, \mathcal{R}_{1}(a, b)$ converges for all $(a, b) \in \mathcal{D}_{3}$.

Remark 2.2. Thus, $\mathcal{R}_{1}(a, a)$ converges on $\hat{\mathcal{C}}$ for all $a$ not pure-imaginary: i.e., $a^{2} \notin(-\infty, 0)$.

Proof: This all follows from the Stieltjes theorem [Lorentzen and Waadeland 92, Theorem 22, page 138].

We can go further, to establish convergence bounds in the following form:

Theorem 2.3. For $(a, b) \in \mathcal{D}_{3}$, so that $b= \pm a$ and $a$ is not pure-imaginary, the convergents to $S$ satisfy

$$
\left|S\left(a^{2}\right)-\frac{p_{n}}{q_{n}}\right|<\frac{2|a|^{2} \sec \theta}{n^{4 /\left(1+\sqrt{1+16|a|^{2} \sec ^{2} \theta}\right)}} .
$$

where $\theta=\min (|\arg (a)|,|\arg (-a)|)$. 
Proof: This follows directly from the Gragg-Warner bounds [Lorentzen and Waadeland 92, page 140] for Stieltjes fractions.

This result can be compared to similar convergence bounds for $\mathcal{R}_{1}(a, a)$, for real $a$, in the companion treatment [Borwein et al. 04a]. The situation is, when $a^{2}=b^{2}$, and $a$ is not pure-imaginary, we do have convergence, but said convergence is "poor," i.e., not geometric (by geometric we mean the error relevant to the $p_{n} / q_{n}$ approximant would be $O\left(\theta^{-n}\right)$ for some real $\left.\theta>1\right)$.

\section{EVEN/ODD FRACTIONS}

For a continued fraction

$$
x:=\frac{a_{1}}{1+\frac{a_{2}}{1+\frac{a_{3}}{1+\frac{a_{4}}{1+} \cdot}}}
$$

a convenient formula with which one may ignite convergence analyses is the classical relation (for $n \geq 1$ )

$$
\frac{p_{n}}{q_{n}}-\frac{p_{n-1}}{q_{n-1}}=(-1)^{n-1} \frac{\prod_{j=1}^{n} a_{j}}{q_{n} q_{n-1}},
$$

with the standard assignments $\left(p_{0}, q_{0}\right):=(0,1)$ and $\left(p_{1}, q_{1}\right):=\left(a_{1}, 1\right)$, and recurrences (for $\left.n \geq 2\right)$ in the form $\left(p_{n}, q_{n}\right)=\left(p_{n-1}, q_{n-1}\right)+a_{n}\left(p_{n-2}, q_{n-2}\right)$. We shall say that any continued fraction converges absolutely if

$$
\sum_{n=1}^{\infty}\left|\frac{p_{n}}{q_{n}}-\frac{p_{n-1}}{q_{n-1}}\right|<\infty
$$

As pointed out in [Lorentzen and Waadeland 92, page 128], if a fraction converges absolutely, then it converges to a finite limit. Similarly, if $x$ has a finite value and $\sum\left|x-p_{n} / q_{n}\right|<\infty$, then $x$ is absolutely convergent, since $\left|p_{n} / q_{n}-p_{n-1} / q_{n-1}\right| \leq\left|x-p_{n} / q_{n}\right|+\left|x-p_{n-1} / q_{n-1}\right|$.

Now, a typical scenario for divergence of $x$ is that the even convergents $p_{2 n} / q_{2 n}$ (to the "even part" of $x$ ) and the odd convergents $p_{2 n+1} / q_{2 n+1}$ (to the "odd" part) approach distinct limits. If, however, the even/odd parts converge absolutely, that is we have both

$$
\sum_{n=0}^{\infty}\left|\frac{p_{2 n+2}}{q_{2 n+2}}-\frac{p_{2 n}}{q_{2 n}}\right|<\infty, \quad \sum_{n=0}^{\infty}\left|\frac{p_{2 n+3}}{q_{2 n+3}}-\frac{p_{2 n+1}}{q_{2 n+1}}\right|<\infty,
$$

then much can be gleaned in regard to convergence of the original fraction $x$, especially if one also knows the
Stern-Stolz construct

$$
\sum_{n=1}^{\infty} \prod_{k=1}^{n}\left|a_{k}\right|^{(-1)^{n-k+1}}
$$

A powerful result in this regard is the following lemma [Lorentzen and Waadeland 92, Lemma 19, page 127], [Jones and Thron 80]:

Lemma 3.1. (Jones-Thron.) If the even/odd parts of $x$ converge absolutely in the sense of (3-2), then $x$ converges if and only if the Stern-Stolz series (3-3) diverges to infinity.

To employ the Jones-Thron result for the Ramanujan fraction, we first write for positive odd integer $M$

$$
\mathcal{R}_{1}(a, b)=\frac{a}{1+\frac{b^{2}}{1+\frac{4 a^{2}}{1+\cdots+\mathcal{S}_{M}(a, b)}}}
$$

where

$$
\mathcal{S}_{M}(a, b):=\frac{M^{2} b^{2}}{1+\frac{(M+1)^{2} a^{2}}{1+\frac{(M+2)^{2} b^{2}}{1+\frac{(M+3)^{2} a^{2}}{1+} \cdot}}} .
$$

We shall focus upon these "tail fractions" $\mathcal{S}_{M}(a, b)$, first dispensing with the Stern-Stolz series issue. Happily, for these tails $\mathcal{S}_{M}$ we always have divergence to infinity of $(3-3)$, as stated in the following theorem:

Theorem 3.2. For any positive odd $M$, the Stern-Stolz series (3-3) for $\mathcal{S}_{M}(a, b)$ diverges to infinity.

Remark 3.3. The companion treatment [Borwein et al. 04a] gives precise, equivalent asymptotics for $M=1$.

Proof: The $n$th summand of the Stern-Stolz series (3-3) is, for $n$ even,

$$
\left|\left(\frac{\Gamma(M / 2+n / 2) \Gamma(M / 2+1 / 2)}{\Gamma(M / 2) \Gamma(M / 2+n / 2+1 / 2)}\right)^{2}(b / a)^{n}\right|,
$$

while for odd index $n$ the summand is

$$
\left|\frac{1}{M b^{2}}\left(\frac{\Gamma(M / 2+n / 2) \Gamma(M / 2+1)}{\Gamma(M / 2+1 / 2) \Gamma(M / 2+n / 2+1 / 2)}\right)^{2}(a / b)^{n-1}\right| .
$$

Now, by the standard Stirling formula, each of the squared-gamma factors is asymptotic to (constant) $\times 1 / n$, so that the sum (3-3) is divergent to infinity. 


$$
\begin{gathered}
c_{M}(n):=-\frac{a^{2} b^{2}(M+2 n-1)^{2}(M+2 n)^{2}}{\left(1+(M+2 n)^{2} b^{2}+(M+2 n+1)^{2} a^{2}\right)\left(1+(M+2 n-2)^{2} b^{2}+(M+2 n-1)^{2} a^{2}\right)}, \\
d_{M}(n):=c_{M}(-M-n+1) .
\end{gathered}
$$

\section{FIGURE 1.}

We now establish exact expressions for the even and odd parts of $\mathcal{S}_{M}(a, b)$ for positive odd $M$. Using standard even/odd decompositions [Lorentzen and Waadeland 92, pages $83-85]$, we have

$$
\begin{aligned}
& \mathcal{S}_{M}^{\text {even }}(a, b)= \\
& \frac{M^{2} b^{2}}{1+(M+1)^{2} a^{2}+\left(1+(M+1)^{2} a^{2}+M^{2} b^{2}\right) F_{M}}, \\
& \mathcal{S}_{M}^{\text {odd }}(a, b)=M^{2} b^{2}+\left(1+(M-1)^{2} a^{2}+M^{2} b^{2}\right) G_{M},
\end{aligned}
$$

where we define

$$
F_{M}:=\frac{c_{M}(1)}{1+\frac{c_{M}(2)}{1+\frac{c_{M}(3)}{1+\frac{c_{M}(4)}{1+} \cdot}}}
$$

and

$$
G_{M}:=\frac{d_{M}(1)}{1+\frac{d_{M}(2)}{1+\frac{d_{M}(3)}{1+\frac{d_{M}(4)}{1+}}}},
$$

with the definitions of $c_{M}(n)$ and $d_{M}(n)$ as in Figure 1.

With a view to Lemma 3.1, our aim is to show that, for certain parameter pairs $(a, b)$, both $\mathcal{S}_{M}^{\text {even }}$ and $\mathcal{S}_{M}^{\text {odd }}$ converge absolutely (and hence to finite values in $\mathcal{C}$ ). In such cases we have $\mathcal{S}_{M}^{\text {even }}=\mathcal{S}_{M}^{\text {odd }}$ as well.

A key function of which we shall make both computational and theoretical use is

$$
c(a, b):=-\frac{a^{2} b^{2}}{\left(a^{2}+b^{2}\right)^{2}},
$$

for this is the asymptotic large- $n$ limit of either $c_{M}(n)$ or $d_{M}(n)$ when $a^{2}+b^{2} \neq 0$. In fact, for $a^{2}+b^{2} \neq 0$ we have

$$
c_{M}(n), d_{M}(n) \sim c(a, b)+O(1 /(M+n)) .
$$

A useful collection of straightforward results is the following:

Lemma 3.4. We have $c(a, b) \notin(-\infty,-1 / 4]$ if and only if $|a| \neq|b|$. In particular, if $a / b=e^{i \phi}$, then $c(a, b)=$ $-(1 / 4) \sec ^{2} \phi$. Finally, if $c(a, b) \notin(\infty,-1 / 4]$, then the two roots of $\omega^{2}-\omega-c(a, b)=0$ are unequal in magnitude.
Proof: If a real $\rho$ has $-\rho \in(\infty,-1 / 4]$, the supposition

$$
a^{2} b^{2} /\left(a^{2}+b^{2}\right)^{2}=\rho
$$

means, with $\rho \geq 1 / 4$,

$$
a / b=\left(\frac{1-2 \rho \pm i \sqrt{4 \rho-1}}{2 \rho}\right)^{1 / 2},
$$

so that $|a / b|=1$. For the converse, with $a=b e^{i \phi}$ (and so the sec-identity is immediate from Definition $(3-5))$ and in the case where $\phi$ is real, we have $c(a, b)=$ $-\frac{1}{4} \sec ^{2} \phi \in(-\infty,-1 / 4]$. Finally, the quadratic roots are $\omega=(1 / 2)(1 \pm \sqrt{1+4 c(a, b)})$. It is a simple geometric observation in the complex plane that $|1-z|=|1+z|$ if and only if $\operatorname{Re}(z)=0$. Thus, the roots can only be equal in magnitude if $c(a, b)$ is real and $\leq-1 / 4$.

\section{4. $\gamma$-FRACTIONS}

With a view to the even/odd decompositions $F_{M}$ and $G_{M}$ of the previous section, we introduce the concept of a $\gamma$-fraction, as

$$
x:=\frac{\gamma_{1}}{1+\frac{\gamma_{2}}{1+\frac{\gamma_{3}}{1+\cdots}}}
$$

where the $\gamma$ elements approach a finite complex limit, say $\gamma_{n} \rightarrow c \in \mathcal{C}$. For our analysis, it is a welcome property of the Ramanujan fraction $\mathcal{R}_{1}$ that both $F_{M}$ and $G_{M}$ of the previous section are, for $a^{2}+b^{2} \neq 0$, gamma-fractions, with $\gamma_{n} \rightarrow c(a, b)$.

It is instructive to consider first the canonical case in which the gamma-fraction $x$ has $\gamma_{n}=c$ for all $n \in \mathcal{Z}^{+}$, whence we have the classical result (see, e.g., [Wall 48]):

Theorem 4.1. Assume that every $\gamma_{n}=c$ with $c \notin$ $(-\infty,-1 / 4)$ (note here the real cut is open). Then $x$ given by (4-1) converges absolutely to the value $r-1$, where $r$ is the larger (in magnitude-see Lemma 3.4) of the roots $(1 \pm \sqrt{1+4 c}) / 2$ of $\omega^{2}-\omega-c=0$. In particular, the convergents of $x$ satisfy

$$
\left|\frac{p_{n}}{q_{n}}-\frac{p_{n-1}}{q_{n-1}}\right|=\left|r(1-s / r)^{2}\right|\left|\frac{s}{r}\right|^{n}
$$


where $s \neq r$ is the other quadratic root (and by Lemma 3.4, $|s|<|r|)$.

Remark 4.2. For $c=-1 / 4$ exactly, the fraction $x$ does converge (to the value $x=-1 / 2$ ), but not absolutely. In fact, $\left|1 / 2+p_{n} / q_{n}\right|=1 /(2 n+2)$ for all $n \geq 0$, and this slow convergence is a hint as to how nonabsolute convergence might occur for some continued fractions $(4-1)$ with $\gamma_{n} \rightarrow c$ more intricately.

Proof: All follows from a closed form for the convergents $p_{n} / q_{n}$ to $x$, namely

$$
\begin{gathered}
p_{n}=c\left(r^{n}-s^{n}\right) /(r-s), \\
q_{n}=\left(r^{n+1}-s^{n+1}\right) /(r-s),
\end{gathered}
$$

and from the fact that $|r|>|s|$.

It turns out that, for any $c \in(-\infty,-1 / 4)$, we have divergence [Wall 48]; for example, with $c:=-1 / 2$ one has

$$
\frac{p_{n}}{q_{n}}=-\frac{1}{\sqrt{2}} \frac{\sin (n \pi / 4)}{\sin ((n+1) \pi / 4)},
$$

whose values oscillate endlessly though $\{0,-1 / 2,-1, \infty\}$. Such observations and Theorem 4.1 completely settle the convergence problem for $\gamma$-fractions with all $\gamma_{n}=c$.

A computational digression is relevant here: it is of interest that the function $c(a, b)$ defined in $(3-5)$ can be used to accelerate rather sluggish situations, in the following way (a similar idea is enunciated in our companion treatment [Borwein et al. 04a] for Gauss continued fractions). We use $(3-5)$ as an approximation to $c_{M}(n)$ for some large $n$, so that, when $a^{2} \neq b^{2}$, the continued fraction $\mathcal{R}_{1}(a, b)$ can be calculated according to the chain starting with (3-4), and $M=1$ - but at a key juncture using the fact that a periodic fraction defined as

$$
x(a, b):=\frac{c(a, b)}{1+\frac{c(a, b)}{1+\frac{c(a, b)}{1+\cdots}}}
$$

is given (via Theorem 4.1) by

$$
x(a, b)=-\frac{a^{2}}{a^{2}+b^{2}} \text { or }-\frac{b^{2}}{a^{2}+b^{2}},
$$

whichever is larger in magnitude. We may therefore attempt to calculate

$$
\mathcal{R}_{1}(a, b)=\frac{a}{1+\frac{b^{2}}{1+4 a^{2}+\left(1+4 a^{2}+b^{2}\right) F_{1}}},
$$

with an approximation presumed accurate for suitably large $n$; namely, we use the finite continued fraction development

$$
F_{1} \approx \frac{c_{1}(1)}{1+\frac{c_{1}(2)}{1+\cdots \frac{c_{1}(n-1)}{1+x(a, b)}}}
$$

That is, in this computational procedure the tail fraction from $c_{1}(n)$ inclusive is replaced by the number $x(a, b)$. This expedient of tail approximation really does improve matters when $|a| \approx|b|$. For example, for $a=b=1$ and the known evaluation $\mathcal{R}_{1}(1,1)=\log 2$ (see [Borwein et al. 04a]), we found that $p_{1000} / q_{1000}$ is correct only to about 3 good decimals for the original continued fraction (1-1); yet, the same amount of work using the even convergents $p_{2000} / q_{2000}$, but also doing the tail-substitution with $x(1,1)=-1 / 2$, yields ten good decimals. Incidentally, rate-bounding in regard to the "oval" theorems in the literature [Lorentzen and Waadeland 92, pages 141146] can be used to effect good bounds on the rate of convergence of such approximations.

We now revert to the theoretical avenue by observing that a relevant set of complex numbers not on a certain real cut can be characterized by

$$
\begin{aligned}
\{c \in \mathcal{C}: c & \notin(-\infty,-1 / 4]\}= \\
& \{c \in \mathcal{C}:|c|<1 / 4\} \cup\{c \in \mathcal{C}:|\arg (c)|<\pi\} .
\end{aligned}
$$

There is overlap in this union, but convenient theorems are possible for each component of said union.

Theorem 4.3. Assume $|c|<1 / 4$ and set $\varepsilon:=1 / 4-|c|$. If in the $\gamma$-fraction $(4-1)$ we have

$$
\left|\gamma_{n}-c\right|<\varepsilon / 2
$$

then $x$ is absolutely convergent, with

$$
\left|\frac{p_{n}}{q_{n}}-\frac{p_{n-1}}{q_{n-1}}\right|<\frac{2}{(1+2 \varepsilon)^{2 n}} .
$$

Proof: Employing the Śleszyński-Pringsheim expedient [Lorentzen and Waadeland 92, page 35] for such bounded elements $\gamma_{n}$, we write the equivalent form

$$
x:=\frac{2 \gamma_{1}}{2+\frac{4 \gamma_{2}}{2+\frac{4 \gamma_{3}}{2+\cdots}}}
$$


and observe for this continued fraction that

$$
\left|q_{n}\right|>2\left|q_{n-1}\right|-(1-2 \varepsilon)\left|q_{n-2}\right| .
$$

Thus, $\left|q_{n}\right|>(1+2 \varepsilon)\left|q_{n-1}\right|$ and so, by (3-1),

$$
\left|\frac{p_{n}}{q_{n}}-\frac{p_{n-1}}{q_{n-1}}\right|<\frac{4^{n}}{2} \frac{\prod_{k=1}^{n} \gamma_{k}}{(1+2 \varepsilon)^{2 n-3}},
$$

and the result follows.

To complete this foray for the set $\{c \notin(-\infty,-1 / 4]\}$, we now establish the following theorem:

Theorem 4.4. Assume $\theta:=|\arg (c)|<\pi$ and that for the $\gamma$-fraction $x(4-1)$ we have

$$
\left|\gamma_{n}-c\right|<h:=\frac{2}{9} \cos ^{2}(\theta / 2) .
$$

Then $x$ is absolutely convergent, with

$$
\left|x-\frac{p_{n}}{q_{n}}\right|<\frac{1}{\sqrt{h}} \frac{|c|+h}{(1+h /(|c|+h))^{n-1}} .
$$

Proof: This follows quickly from the parabola-sequence theorem [Lorentzen and Waadeland 92, Theorem 21, pages 136-137], with the multiplier assignment $g_{k}:=1 / 3$.

Now we have the central result of the present treatment:

Theorem 4.5. For $|a| \neq|b|$, the Ramanujan fraction $\mathcal{R}_{1}(a, b)$ converges on $\hat{\mathcal{C}}$.

Proof: By Lemma 3.4, $|a| \neq|b|$ implies $c(a, b) \notin$ $(-\infty,-1 / 4]$. By Lemma 3.1 and Theorems 4.3 and 4.4, and by the observation that for sufficiently large odd $M$ the bounds on $\left|\gamma_{n}-c(a, b)\right|$ in the two stated theorems are indeed met either for $\gamma_{n}:=c_{M}(n)$ or for $\gamma_{n}:=d_{M}(n)$, we have absolute convergence of the even/odd parts of $\mathcal{S}_{M}$; hence, convergence of the original fraction $\mathcal{R}_{1}(a, b)$.

Corollary 4.6. $\mathcal{D}_{1} \subseteq \mathcal{D}_{0}$, that is, $\mathcal{R}_{1}(a, b)$ converges on $\hat{\mathcal{C}}$ if $|a| \neq|b|$ or $a^{2}=b^{2}$ with a not pure-imaginary.

Proof: This follows from Theorems 2.1 and 4.5.

\section{DIVERGENCE}

A special case of divergence of $\mathcal{R}_{1}$ runs as follows:

Theorem 5.1. If a is pure-imaginary, that is $a^{2} \in$ $(-\infty, 0)$, then the fraction $\mathcal{R}_{1}(a, a)$ diverges. In particular, $\mathcal{R}_{1}(i, i)$ diverges.
Proof: We have in this case

$$
c_{1}(n)=-\frac{1}{4}+\frac{1}{16 n^{2}}\left(\frac{1}{a^{2}}-1\right)+\cdots .
$$

Now, the Jacobsen-Masson theory (see [Lorentzen and Waadeland 92, Theorem 32, page 159] and references therein) shows that, if negative-real fraction elements $c_{1}(n)$ are eventually less than $-\frac{1}{4}-\frac{r}{16 n^{2}}$ for some real $r>1$, then the fraction diverges. Thus, $\mathcal{S}_{1}^{\text {even }}(a, a)$ diverges, and so $\mathcal{R}_{1}(a, a)$ cannot converge. (Similarly, the odd part $\mathcal{S}_{1}^{\text {odd }}$ diverges.)

In attempting to establish divergence for other parameter pairs, in particular the cases $a=b i$, we developed means to combine computation and theory and prove inequality of the even/odd parts, even though both parts often themselves converge. The technique starts with the assumption of a fraction (4-1), but not a gamma-fraction, as $\gamma_{n} \rightarrow \infty$; instead,

$$
\gamma_{n}:=\left(n+\delta_{n}\right)^{2},
$$

which assignment-when we know $c_{1}(n)$ and $d_{1}(n)$ for cases $a=b i$-implicitly defines the pertubations $\delta_{n}$. An attractive recurrence-transformation results if we define $\rho_{n}$ implicitly by

$$
q_{n}=\rho_{n} \prod_{j=1}^{n+1}\left(j+\delta_{j}\right),
$$

while the usual recurrence $q_{n}=q_{n-1}+\gamma_{n} q_{n-2}$ for $q_{0}=$ $q_{1}=1$ yields

$$
\rho_{n}=\frac{\rho_{n-1}+\left(n+\delta_{n}\right) \rho_{n-2}}{n+1+\delta_{n+1}} .
$$

In turn, we have an exact formula

$$
\Delta_{n}:=\frac{p_{n}}{q_{n}}-\frac{p_{n-1}}{q_{n-1}}=\frac{(-1)^{n}}{\rho_{n} \rho_{n-1}} \frac{1}{n+1+\delta_{n+1}} .
$$

For suitably bounded $\left|\delta_{n}\right|$ and for $\rho_{n}$ confined to, say, a circle in the proper right half-plane, the series for the fraction $x=\sum_{n \geq 1} \Delta_{n}$ is convergent; moreover, we can establish bounds on the error relevant to the $p_{n} / q_{n}$ approximant. Our technique, then, is to calculate the even/odd parts to some level $n$ and bound the error such that we know rigorously the inequality of said even/odd parts.

An exemplary application of this computationaltheoretical fusion is the following:

Theorem 5.2. Both $\mathcal{R}_{1}(1, i)$ and $\mathcal{R}_{1}\left(e^{i \pi / 4}, e^{-i \pi / 4}\right)$ diverge. 
Remark 5.3. The second case of the theorem contradicts previous literature claims that convergence occurs for $\operatorname{Re}(a), \operatorname{Re}(b)>0$; see [Borwein et al. 04a].

Proof: For $(a, b)=(1, i)$ we have

$$
S_{1}^{e v e n}(1, i)=\frac{-1}{5+4 F_{1}}
$$

where

$$
F_{1}:=\frac{c_{1}(1)}{1+\frac{c_{1}(2)}{1+\frac{c_{1}(3)}{1+} \cdot}}
$$

with, here,

$$
c_{1}(n):=\frac{n(2 n+1)^{2}}{4(n+1)}
$$

(and note that relation (3-6) does not apply, as $a^{2}+b^{2}=$ $0)$. This $F_{1}$ does converge to a finite value according to the above analysis involving the $\rho_{n}$ or to the "parabola" theorem [Lorentzen and Waadeland 92, Theorem 20, page 130]. In this particular case, $(a, b)=(1, i)$, the error analysis can be simplified. We have $n^{2}<c_{1}(n)<$ $n^{2}+1 / 4$, so the recursion $q_{n}=q_{n-1}+c_{1}(n) q_{n-2}>$ $q_{n-1}+n^{2} q_{n-2}$ tells us that, in fact, $q_{n} \geq(n+1) ! / 2$. Thus, we have (the first inequality here is allowed when all fraction elements are positive real)

$$
\left|F_{1}-\frac{p_{n}}{q_{n}}\right| \leq\left|\frac{\prod_{j=1}^{n} c_{1}(j)}{q_{n} q_{n-1}}\right|<\frac{d}{n+1},
$$

for a positive constant $d$. The convergence is "slow" and nonabsolute, but one may use this convergence bound together with computation up to appropriate $n$ to establish

$$
\mathcal{S}_{1}^{\text {even }}(1, i) \in[-0.15,-0.14] .
$$

On the other hand, one may show in similar fashion that

$$
S_{1}^{\text {odd }}(1, i)=-1+\frac{-1}{1+\frac{c_{1}(-2)}{1+\frac{c_{1}(-3)}{1+\frac{\left.c_{(}-4\right)}{1+}}}} \in[-1.5,-1.4],
$$

so $\mathcal{S}_{1}(1, i)$ is shown to have distinct even/odd parts. Since $\mathcal{R}_{1}(a, b)=a /\left(1+S_{1}(a, b)\right)$, we thus see that the even/odd parts of $\mathcal{R}_{1}$ are known as

$$
\begin{gathered}
\mathcal{R}_{1}^{\text {even }}(1, i) \approx 1.167, \\
\mathcal{R}_{1}^{\text {odd }}(1, i) \approx-2.38 \ldots,
\end{gathered}
$$

both provably correct to the implied precision; thus, $\mathcal{R}_{1}(1, i)$ diverges.

For $(a, b)=\left(e^{i \pi / 4}, e^{-i \pi / 4}\right)$ the parabola theorem applies with

$$
c_{1}(n):=\frac{2 n^{2}(2 n+1)^{2}}{-2-i+(4-4 i) n+8 n^{2}}, \quad d_{1}(n):=c_{1}(-n) ;
$$

so, both $F_{1}$ and $G_{1}$ converge to finite values. This convergence can also be shown via the aformentioned definition $\gamma_{n}:=\left(n+\delta_{n}\right)^{2}$ with

$$
\delta_{n}=\sqrt{i / 8}+O\left(1 / n^{2}\right) .
$$

The computation-bounding technique for, say, $n=10^{5}$ and a suitable error bound (we omit the details on bounding of $\rho_{n}$ ) yields

$$
\begin{aligned}
& \mathcal{R}_{1}^{\text {even }}\left(e^{i \pi / 4} \cdot e^{-i \pi / 4}\right) \approx 0.8185+0.867 i, \\
& \mathcal{R}_{1}^{\text {odd }}\left(e^{i \pi / 4} \cdot e^{-i \pi / 4}\right) \approx-0.103+0.583 i,
\end{aligned}
$$

both approximations correct to the implied precision. Thus, $\mathcal{R}_{1}$ does not converge for the given parameter pair.

Such isolated divergence results, together with extensive computations, have led us to the following conjecture. (Again, a separate work has resolved a good deal of conjecture; in particular, Conjectures 5.4 and 5.5, and the implicit conjecture in Remark 5.6, are now proven [Borwein et al. 04b]).

Conjecture 5.4. $\mathcal{D}_{0}=\mathcal{D}_{1}$. Equivalently, given Corollary 4.6. $\mathcal{R}_{1}(a, b)$ diverges if $a / b=e^{i \phi}$ with $\cos ^{2} \phi \neq 1$.

We have been able to refine Conjecture 5.4-which would completely settle the convergence question for the Ramanujan fraction - down to the following (experimentally motivated) form, amounting to a dynamical equivalent for divergence:

Conjecture 5.5. For complex nonzero $a$ and real $\phi$ with $\cos ^{2} \phi \neq 1$, or $a \in \mathcal{I}$ and $\cos ^{2} \phi=1$, and any complex initial values $\left(r_{0}, r_{1}\right)$, the sequence $\left(r_{n}\right)$ determined by the recurrence $(n>1)$,

$$
\begin{aligned}
& r_{n}=\frac{1}{a(n+1 / 2)} r_{n-1}+\frac{n^{2}}{n^{2}-1 / 4} r_{n-2}, \quad n \text { even, } \\
& r_{n}=\frac{1}{a(n+1 / 2)} r_{n-1}+\frac{n^{2} e^{2 i \phi}}{n^{2}-1 / 4} r_{n-2}, \quad n \text { odd },
\end{aligned}
$$

is bounded in $\mathcal{C}$. 
Remark 5.6. One could also posit that a recurrence

$$
\rho_{n}=\frac{\rho_{n-1}+n \omega_{n} \rho_{n-2}}{n+1},
$$

with $\omega_{n}=a^{2}$ or $\omega_{n}=a^{2} e^{2 i \phi}$ as $n$ is even/odd respectively, has $\rho_{n}=O\left(a^{n} / \sqrt{n}\right)$, yielding an equivalent analysis. The advantage of the particular recurrence form in Conjecture 5.5 is the simple goal of boundedness of the $\left|r_{n}\right|$, while the advantage of the $\rho$-recurrence suggested here is that the algebra is less recondite. We note that Conjecture 5.5 has been indirectly settled, via Theorems 5.1 and 5.2 (and the analysis in the following Theorem 5.6), for the cases $a$ pure-imaginary and $\phi=0$, $(a, \phi)=(1, \pi / 2)$, and $(a, \phi)=(\sqrt{i},-\pi / 2)$. Also, though we believe the boundedness of the $r_{n}$ is independent of initial values, we could, if necessary, posit a conjecture having $r_{0}:=1 / \Gamma(3 / 2)$ and $r_{1}:=1 /(a \Gamma(5 / 2))$ (or for the alternative $\rho$ sequence, $\rho_{0}:=1$ and $\left.\rho_{1}:=1 / 2\right)$, for such initial values are consistent with $q_{0}=q_{1}:=1$ for the original fraction.

The fascinating recurrence in Conjecture 5.5- or its various equivalent recurrences as in Remark 5.6-give rise to the next theorem:

Theorem 5.7. Conjecture 5.5 implies Conjecture 5.4, i.e., that $\mathcal{D}_{0}=\mathcal{D}_{1}$.

Proof: Let $p_{n} / q_{n}$ be the convergents to the fraction

$$
\mathcal{S}_{1}(a, b):=\frac{b^{2}}{1+\frac{4 a^{2}}{1+\frac{9 b^{2}}{1+\frac{16 a^{2}}{1+} \cdot}}},
$$

where $a / b=e^{i \phi}$ with real $\phi$ and $\cos ^{2} \phi \neq 1$. We have $q_{0}=q_{1}=1$. Now define

$$
r_{n}:=\frac{q_{n}}{a^{n} \Gamma(n+3 / 2)},
$$

so that the $r_{n}$ satisfy the recurrences of Conjecture 5.5. For the $\mathcal{S}_{1}$ fraction, we have, for $n$ even, via relation $(3-1)$,

$$
\Delta_{n}:=\left|\frac{p_{n}}{q_{n}}-\frac{p_{n-1}}{q_{n-1}}\right|=\frac{n !^{2}}{\Gamma(n+3 / 2) \Gamma(n+1 / 2)}\left|\frac{a e^{i n \phi}}{r_{n} r_{n-1}}\right| .
$$

Thus, by Conjecture $5.5, \Delta_{n}$ is thus bounded below, and so $\mathcal{S}_{1}$, and hence $\mathcal{R}_{1}$, is divergent.
Conjecture 5.5-which would completely settle the convergence problem for the Ramanujan fraction - is motivated by extensive numerical experiments: the $r_{n}$ of said conjecture appear to be bounded (alternatively, the $\rho_{n} / a^{n}$ decay like $1 / \sqrt{n}$ ) in every case we have studied. One thing we can say at this juncture: the theory of Gill on Möbius transforms [Gill 73] implies that, for $a / b=e^{i \phi}$ with $\cos ^{2} \phi \neq 1$, then both even and odd parts of $\mathcal{R}_{1}(a, b)$ do converge. (Indeed, we saw two manifestations of this in Theorem 5.2.) We are saying via our conjectures that such even/odd parts should converge to distinct limits. Thus, there is a kind of "bifurcation" for $a / b=e^{i \phi}$ with $\cos ^{2} \phi \neq 1$. For the parameter instances $a^{2}=b^{2}$ for $b \in \mathcal{I}$, it turns out that both even/odd parts of $\mathcal{R}_{1}(a, b)$ are always bifurcated or in some way chaotic. The resolutions of these various conjectures and ideas follow the spirit of Theorem 5.7 as applied to specific recurrence relations [Borwein et al. 04b].

\section{AGM RELATION REVISITED}

The remarkable AGM relation (1-2) that motivated both this and the companion [Borwein et al. 04a] treatments can now be put in perspective:

Theorem 6.1. If $a / b \in \mathcal{H}$ then each of the three fractions

$$
\mathcal{R}_{1}(a, b), \mathcal{R}_{1}(b, a), \text { and } \mathcal{R}_{1}((a+b) / 2, \sqrt{a b})
$$

converges on $\hat{\mathcal{C}}$.

Proof: For $a / b \in \mathcal{H}$ none of the relevant parameter pairs enjoy equal magnitudes, so Theorem 4.5 settles the issue.

It is fascinating that, in spite of Theorem 6.1 -and as suggested in the abstract - there are parameter pairs $(a, b)$ where all three fractions converge and yet the AGM relation (1-2) is false. For example,

$$
\mathcal{R}_{1}(2 i, 1)+\mathcal{R}_{1}(1,2 i) \neq 2 \mathcal{R}_{1}(1 / 2+i, 1+i),
$$

which can be gleaned easily via some computation and the relatively strong bounds of Theorem 4.4.

Conjecture 6.2. For $a / b \in \mathcal{H}$ the $A G M$ relation (1-2) holds on $\hat{\mathcal{C}}$ (with, as we know, all fractions converging on $\hat{\mathcal{C}}$ ).

In regard to Theorem 6.1 and Conjecture 6.2, one must take care to observe certain anomalies. For example, it 
turns out that $\mathcal{R}_{1}(a, b)$ converges to infinity when

$$
a:=i \frac{\Gamma^{2}(1 / 4)}{4 \pi^{3 / 2}}, \quad b:=i \frac{\Gamma^{2}(1 / 4)}{4 \pi^{3 / 2} \sqrt{2}}
$$

even though $a / b \in \mathcal{H}$; here Conjecture 6.2 remains intact, in the sense that the AGM relation for this pair $(a, b)$ then reads $\infty=\infty$. Note that for this peculiar parameter pair (and certain others) the fraction

$$
\mathcal{S}_{1}(a, b):=\frac{b^{2}}{1+\frac{4 a^{2}}{1+\frac{9 b^{2}}{1+\frac{16 a^{2}}{1+} \cdot}}}
$$

actually converges to the finite value -1 . Such singularities in the AGM relation can also be inferred from the sech identities (3-1) and (3-2) in the treatment [Borwein et al. 04a] that reveal the possibility of infinitely many poles in the summation.

We believe it very likely that Conjecture 6.2 would follow from careful examination of the analyticity properties (in $\eta, a, b)$ of the aforementioned sech series and the corresponding properties for the continued fractions with $|a| \neq|b|$.

\section{OPEN ISSUES}

- We still do not know an exact evaluation - in the sense, say, of closed forms as in [Borwein et al. 04a] for $\mathcal{R}_{1}(a, a)$ with certain $a$-for unequal $a$ and $b$; except, as we state in Section 6 , we do know some $(a, b)$ with $\mathcal{R}_{1}(a, b)=\infty$.
- Since the conjectures of Section 5 have been resolved in a separate treatment [Borwein et al. 04b], there remains Conjecture 6.2, which is open. Aside from the difficult problem of correctly analyzing sech identities (see end of Section 6), there is also the dilemma of what points on the closure of $\mathcal{H}$ are valid AGM points.

\section{ACKNOWLEDGMENTS}

We thank Bruce Berndt, Joseph Buhler, Greg Fee, William Jones, Lisa Lorentzen, and Stephen Rayhawk for useful discussions and observations. The reserach of the first author was supported by NSERC, the Canada Foundation for Innovation, and the Canada Research Chair Program.

\section{REFERENCES}

[Borwein et al. 04a] J. Borwein, R. Crandall, and G. Fee. "On the Ramanujan AGM Fraction, I: The RealParameter Case" Exper. Math. 13:3 (2004), 275-286.

[Borwein et al. 04b] D. Borwein, J. Borwein, R. Crandall, and R. Mayer. "On the Dynamics of Certain Recurrence Relations." Preprint, 2004. Available from World Wide Web (http://www.cecm.sfu.ca/preprints).

[Gill 73] J. Gill. "Infinite Compositions of Mobius Transforms." Trans. Amer. Math. Soc. 176 (1973), 479-487.

[Jones and Thron 80] W. Jones and W. Thron. Continued Fractions: Analytic Theory and Applications. Reading, MA: Addison-Wesley, 1980.

[Lorentzen and Waadeland 92] L. Lorentzen and H. Waadeland. Continued Fractions With Applications. Amsterdam: North-Holland, 1992.

[Wall 48] H. S. Wall. Analytic Theory of Continued Fractions. New York: Van Nostrand, 1948.

J. Borwein, Faculty of Computer Science, Dalhousie University, Halifax, Nova Scotia B3H 1W5, Canada (jborwein@cs.dal.ca)

R. Crandall, Center for Advanced Computation, Reed College, Portland, OR 97202 (crandall@reed.edu)

Received June 17, 2003; accepted August 29, 2003. 